\title{
Holomorphic approximation of CR functions on tubular submanifolds of $\mathbb{C}^{2}$
}

\author{
by André Boivin* (London,Ont.) and Roman Dwilewicz** (Warszawa)
}

\begin{abstract}
The purpose of this paper is to take a closer look at uniform semi-global (i.e. on compact subsets) holomorphic approximation of $\mathrm{CR}$ functions on tubular submanifolds in $\mathbb{C}^{2}$.
\end{abstract}

$\S$ 0. Introduction. One of the main problems in the theory of CauchyRiemann (CR) functions is the problem of their approximation by holomorphic functions. The local approximation problem was completely solved by Baouendi and Trèves [BT] in 1981. The global approximation problem was solved only in special cases like totally real submanifolds (see Nunemacher [N1]) and generic CR submanifolds of $\mathbb{C}^{n}$ which satisfy additional assumptions (see $[\mathrm{DG}],[\mathrm{N} 2],[\mathrm{S}]$ ). For a review article on this subject see [D].

The global holomorphic approximation problem in complete generality seems to be very difficult. For instance, even the case of CR functions defined on boundaries of pseudoconvex domains is not very well understood (see $[\mathrm{DF}],[\mathrm{FN}]$ ).

The purpose of this paper is to take a closer look at uniform global or semi-global holomorphic approximation of CR functions on tubular submanifolds in $\mathbb{C}^{2}$. By uniform semi-global approximation or almost uniform approximation on a manifold $M$, we mean uniform approximation on the compact subsets of $M$. We prove the following results.

THEOREM 0.1. Let $M$ be a tubular submanifold of $\mathbb{C}^{2}$ over a compact connected manifold $N \subset \mathbb{R}^{2}$. Then any $C R$ function on $M$ can be almost uniformly approximated by holomorphic polynomials.

TheOREM 0.2. Let $M$ be a tubular submanifold of $\mathbb{C}^{2}$ over a connected

1991 Mathematics Subject Classification: Primary 32E30; Secondary 32F25.

* Research supported in part by the Natural Sciences and Engineering Research Council of Canada.

** Partially supported by Grant RP.I.10 of the Polish Ministry of National Education. 
embedded manifold $N \subset \mathbb{R}^{2}$. If $N$ is a curve, then additionally assume that on any compact arc there are only finitely many inflexion points. Then any CR function on $M$ can be almost uniformly approximated by holomorphic polynomials.

COROLlary 0.3. Let $M$ be a tubular submanifold of $\mathbb{C}^{2}$ over an embedded manifold $N \subset \mathbb{R}^{2}$. Moreover, assume that any non-compact 1-dimensional component of $N$ satisfies the assumption of Theorem 0.2. Then any $C R$ function on $M$ can be almost uniformly approximated by holomorphic functions defined in a neighbourhood of $M$ in $\mathbb{C}^{2}$.

Remark. The approximating polynomials in Theorem 0.1 and 0.2 are explicitly constructed in terms of derivatives at some point of the approximated function or its extension.

The restriction on the curve $N$ in Theorem 0.2 concerning the inflexion points comes from Kazlow's extension theorem $[\mathrm{K}]$ which we use in the proof.

The proofs of the above theorems cannot be immediately extended to tubular CR manifolds in $\mathbb{C}^{n}, n \geq 3$; additional considerations are needed. Some approximation results for higher dimensional tubular CR manifolds will be published by the authors in a subsequent paper.

The organization of the paper is as follows. In $\S 1$ we give some notation and definitions. In $\S 2$ an immediate consequence of the paper [DG] for tubular manifolds is formulated. In $\S 3$ some geometrical lemmas are proved and finally in $\S 4$ the proofs of the theorems are given.

The authors would like to thank Professor Józef Siciak for suggesting the use of Mittag-Leffler approximations in starlike shaped domains.

$\S 1$. Notation and definitions. All manifolds considered here are of class $C^{\infty}$ embedded into $\mathbb{R}^{n}$ or $\mathbb{C}^{n}$, and all the functions will be of class $C^{\infty}$ unless otherwise stated.

Let $\left(z_{1}, \ldots, z_{n}\right)$ be the complex coordinates in $\mathbb{C}^{n}$, and let $z_{k}=x_{k}+i y_{k}$, $x_{k}=\operatorname{Re} z_{k}, y_{k}=\operatorname{Im} z_{k}, k=1, \ldots, n$.

By a Cauchy-Riemann $(C R)$ submanifold $M$ of $\mathbb{C}^{n}$ we mean a submanifold such that

$$
\ell=\ell(p)=\operatorname{dim}_{\mathbb{C}} \mathbb{C} T_{p}(M) \cap H_{p}^{0,1}\left(\mathbb{C}^{n}\right)
$$

is constant on $M$, where $\mathbb{C} T(M)$ is the complexified tangent bundle to $M$ and $H^{0,1}\left(\mathbb{C}^{n}\right)$ is the tangent bundle to $\mathbb{C}^{n}$ of vectors of type $(0,1)$.

By a $C R$ function on $M$ we mean a smooth function $f: M \rightarrow \mathbb{C}$ which is annihilated by all the sections of $\left.\mathbb{C} T(M) \cap H^{0,1}\left(\mathbb{C}^{n}\right)\right|_{M}$.

By a tubular submanifold $M$ of $\mathbb{C}^{n}$ we mean a submanifold of the form

$$
M=N \times i \mathbb{R}^{n} \subset \mathbb{R}^{n} \times i \mathbb{R}^{n}=\mathbb{C}^{n}
$$


where $N$ is a smooth embedded submanifold of $\mathbb{R}^{n}$. Sometimes we shall denote $M$ by $\tau(N)$, a tube over $N$. It is very easy to check that tubular submanifolds are automatically CR submanifolds of $\mathbb{C}^{n}$.

Let $S$ be a subset of $\mathbb{R}^{n}$ or of $\mathbb{C}^{n}$. The convex hull of $S, \operatorname{ch}(S)$, is the smallest convex set containing $S$. Notice that for tubular manifolds $\tau(N) \subset \mathbb{C}^{n}, N \subset \mathbb{R}^{n}$, we have

$$
\operatorname{ch}(\tau(N))=\tau(\operatorname{ch}(N)) .
$$

For a submanifold $N \subset \mathbb{R}^{2}$ define the almost convex hulls of $N$ and $\tau(N)$ as

$$
\operatorname{ach}(N)=N \cup \operatorname{Int}(\operatorname{ch}(N)), \quad \operatorname{ach}(\tau(N))=\tau(N) \cup \operatorname{Int}(\tau(\operatorname{ch}(N))) .
$$

where the interior Int is taken in $\mathbb{R}^{2}$ and $\mathbb{C}^{2}$, respectively.

We shall denote the boundary of a set $S$ by $\operatorname{bd}(S)$.

A subset $S$ of $\mathbb{R}^{n}$ or $\mathbb{C}^{n}$ is said to be starlike with respect to a point $p \in S$ if every point of $S$ can be joined to $p$ by a line segment contained in $S$. If, moreover, $S \Subset \operatorname{Int} S_{r}$ for any $0<r<1$, where

$$
S_{r}=\left\{p+\frac{1}{r}(q-p): q \in S\right\},
$$

we say that $S$ is strictly starlike with respect to $p$.

$\S 2$. Some consequences of the paper [DG] for tubular manifolds. First we give a definition of the $\mathcal{R}$-property which plays a major role in $[\mathrm{DG}]$.

Definition 2.1. We say that a CR submanifold $M$ of $\mathbb{C}^{n}$ has the $\mathcal{R}$ property if there exists a smooth $n$-real-dimensional distribution $L: M \rightarrow$ $T(M)$ and a smooth $\mathrm{CR}$ matrix-valued function $A: M \rightarrow \operatorname{GL}(n, \mathbb{C})$ such that

$$
|\operatorname{Im} v|<|\operatorname{Re} v| \quad \text { for } v \in A(p) L_{p}, v \neq 0, p \in M,
$$

where $\mid$ denotes the canonical norm in $\mathbb{C}^{n}$.

Roughly speaking, at each point $p \in M$, the matrix $A(p)$, as a $\mathbb{C}$-linear mapping of $\mathbb{C}^{n}$ into $\mathbb{C}^{n}$, "turns" a real subspace $L_{p} \subset T_{p}(M) \subset \mathbb{C}^{n}$ so that the image is "close" to $\mathbb{R}^{n}$.

Notice that tubular submanifolds in $\mathbb{C}^{n}$ have the $\mathcal{R}$-property. It is enough to take $L(p)=i \mathbb{R}^{n} \subset T_{p}(M)$ and $A(p)=i I$, where $I$ is the unit matrix.

Consequently, the main theorem of $[\mathrm{DG}]$, when applied to tubular manifolds, reads:

THEOREM 2.1. Let $M$ be a connected tubular manifold in $\mathbb{C}^{n}$. Then there exists a neighbourhood $\Omega$ of $M$ in $\mathbb{C}^{n}$ with the following property: for any strongly pseudoconvex domain $D, D \Subset \Omega$, with smooth boundary, any smooth $C R$ function $f: M \rightarrow \mathbb{C}$ can be uniformly approximated on $M \cap \bar{D}$ by functions holomorphic on neighbourhoods of $\bar{D}$. 
If the base $N$ of the tubular manifold $M$ is arbitrary, then, of course, it is not clear what are the properties of the neighbourhood $\Omega$ in Theorem 2.1, and in particular $\Omega$ may not contain non-trivial strongly pseudoconvex domains, for example one which would contain $N$. The existence of such a domain $D$ is, however, clear when $N$ bounds a strictly convex domain in $\mathbb{R}^{n}$.

In the proof of Theorem 2.1, an integral formula of Baouendi-Trèves [BT] for local holomorphic approximation of $\mathrm{CR}$ functions is used. Using the same formula, a result on global holomorphic approximation of CR functions on tubular manifolds (with some restriction on the growth at infinity) will be published by Chirka [C].

$\S 3$. Some geometrical lemmas. In this section we prove some technical geometrical lemmas which we need in the proof of the main theorems.

Lemma 3.1. Let $N$ be a smooth Jordan (i.e. simple and closed) curve in $\mathbb{R}^{2}$ which bounds an open domain $V$. For any point $p \in V$ there exists a Jordan curve $C$ with the following properties:

(i) $C$ is smooth except maybe at an at most countable number of points.

(ii) $C \backslash(N \cap \operatorname{bd}(\operatorname{ch}(N))) \subset \operatorname{Int}(\operatorname{ch}(N))$.

(iii) The domain $W$ bounded by $C$ is strictly starlike with respect to $p$.

(iv) There exists a neighbourhood in $N$ of $N \cap \mathrm{bd}(\mathrm{ch}(N))$ that is contained in $C$.

(v) $V \subset W$.

Proof. Notice that $\operatorname{bd}(\operatorname{ch}(N))$ is a $C^{1}$ Jordan curve. Fix $p$ in $V$. Of course $\operatorname{ch}(N)$ is strictly starlike with respect to $p$.

Take a smooth parametrization $\varphi:[0,1] \rightarrow N$ of $N, \varphi=\left(\varphi_{1}, \varphi_{2}\right)$, $\varphi(0)=\varphi(1)$. The set

$$
A=\{t \in[0,1]: \varphi(t) \in N \cap \operatorname{bd}(\operatorname{ch}(N))\}
$$

is compact and consequently $B=[0,1] \backslash A$ is open in $[0,1]$ and is a sum of open (in $[0,1]$ ) intervals

$$
B=\bigcup_{n=1}^{\infty} I_{n} .
$$

Take any interval $I_{n}$. Without loss of generality we can assume that $I_{n}=$ $\left(a_{n}, b_{n}\right)$. Take the corresponding arc $J_{n}=\varphi\left(\left(a_{n}, b_{n}\right)\right)$, and notice that $J_{n}$ lies in $\operatorname{ch}(V)$.

The piece of the boundary of $\operatorname{ch}(V)$ between $\varphi\left(a_{n}\right)$ and $\varphi\left(b_{n}\right)$ is a segment of the straight line $L_{n}$ passing through $\varphi\left(a_{n}\right)$ and $\varphi\left(b_{n}\right)$. Take a continuous family $L_{n}(s), s \in \mathbb{R}$, of straight lines parallel to $L_{n}$ and such that the distance between $L_{n}$ and $L_{n}(s)$ is equal to $|s|$, and moreover $L_{n}(s) \neq L_{n}(-s)$. 
Changing the parameter $s$ if necessary, we can assume that $L_{n}(s) \cap V \neq \emptyset$ for sufficiently small positive $s$, and $L_{n}(s) \cap V=\emptyset$ for $s \leq 0$.

For any $\varepsilon_{n}>0$ there exists $s_{n}>0$ such that for $0<s \leq s_{n}$,

$$
L_{n}(s) \cap J_{n} \Subset \varphi\left(\left(a_{n}, a_{n}+\varepsilon_{n}\right) \cup\left(b_{n}-\varepsilon_{n}, b_{n}\right)\right) .
$$

Take

$$
\begin{aligned}
& \alpha_{n}=\sup \left\{t \in\left(a_{n}, a_{n}+\varepsilon_{n}\right): \varphi(t) \in L_{n}\left(s_{n} / 2\right)\right\}, \\
& \beta_{n}=\inf \left\{t \in\left(b_{n}-\varepsilon_{n}, b_{n}\right): \varphi(t) \in L_{n}\left(s_{n} / 2\right)\right\} .
\end{aligned}
$$

Define a new curve $C$ by the following parametrization $\Psi:[0,1] \rightarrow C$ :

$$
\begin{aligned}
\Psi(t)= & \varphi(t) \quad \text { for } t \in[0,1] \backslash \bigcup_{n=1}^{\infty}\left(\alpha_{n}, \beta_{n}\right), \\
\Psi(t)= & \text { the linear parametrization of the segment of } L_{n}\left(s_{n} / 2\right) \\
& \text { between } \varphi\left(\alpha_{n}\right), \varphi\left(\beta_{n}\right) \text { for } t \in\left(\alpha_{n}, \beta_{n}\right) .
\end{aligned}
$$

It is obvious that $\Psi$ is continuous on $[0,1]$ and smooth except maybe at $\alpha_{n}$, $\beta_{n}, n=1,2, \ldots$. Also it is clear that if $\varepsilon_{n}, n=1,2, \ldots$, are sufficiently small, then the domain $W$ bounded by $C$ is strictly starlike with respect to $p$.

The other properties of $C$ follow immediately from the construction. The proof of the lemma is complete.

Let $N$ be an arbitrary curve in $\mathbb{R}^{2}$ parametrized continuously by $\varphi$ : $[0,1] \rightarrow N$, where $\varphi$ is smooth on $(0,1)$. Set $\varphi(0)=p_{0}, \varphi(1)=p_{1}$. Assume also that $\operatorname{Int}(\operatorname{ch}(N)) \neq \emptyset$. With these assumptions, we formulate the following two lemmas:

LEMMA 3.2. If $p_{0}, p_{1} \in \operatorname{Int}(\operatorname{ch}(N))$, then for any $p \in \operatorname{Int}(\operatorname{ch}(N))$ there exists a Jordan curve $C$ which satisfies the conditions (i)-(iv) of Lemma 3.1, and moreover $N \subset C \cup W$, where $W$ is the domain bounded by $C$.

LEMMA 3.3. If $p_{0}, p_{1}$ do not both belong to $\operatorname{Int}(\operatorname{ch}(N))$, then for any $p \in$ $\operatorname{Int}(\operatorname{ch}(N))$ and any $\varepsilon>0$ there exists a Jordan curve $C$ which satisfies (i)(iii) of Lemma 3.1 together with $\varphi([\varepsilon, 1-\varepsilon]) \subset C \cup W$, and a neighbourhood in $N$ of $\varphi([\varepsilon, 1-\varepsilon]) \cap \operatorname{bd}(\operatorname{ch}(N))$ is contained in $C$.

The proof of Lemma 3.2 is exactly the same as that of Lemma 3.1, and the proof of Lemma 3.3 requires only a slight modification; we omit them here.

\section{$\S$ 4. Proof of Theorems 0.1 and 0.2}

Proof of Theorem 0.1. Under the assumptions of the theorem we have only two possibilities for the base $N: N$ is a either point or a Jordan curve. 
The first case is trivial and follows immediately from the Stone-Weierstrass theorem. So it is enough to consider the second case.

Take any smooth $\mathrm{CR}$ function $f: M \rightarrow \mathbb{C}$. There is a smooth extension $F$ of $f$ to the tube $\tau(\bar{V})$ that is holomorphic in $\tau(V)$, where $V$ is the domain bounded by $N$. The last statement immediately follows from $[\mathrm{AH}$, Section 4.5]. By Bochner's tube theorem, $F$ extends holomorphically to the convex hull $\operatorname{ch}(\tau(V))$. We do not know anything about the boundary behaviour of $F$ on the latter set, but using Lemma 3.1 we can construct a curve $C$ bounding a strictly starlike domain $W$ that satisfies all the properties (i)-(v) of the lemma, and such that $F$ is smooth on the compact set $W \cup C$. For simplicity we can assume that $W$ is strictly starlike with respect to the origin.

For each $r>0$ define

$$
F_{r}\left(z_{1}, z_{2}\right)=F\left(r z_{1}, r z_{2}\right), \quad\left(z_{1}, z_{2}\right) \in \Omega_{r},
$$

where

$$
\Omega_{r}=\left\{\left(z_{1}, z_{2}\right) \in \mathbb{C}^{2}:\left(r z_{1}, r z_{2}\right) \in \tau(W)\right\} .
$$

By the properties of $W$, we have $\tau(\bar{V}) \Subset \Omega_{r}$ for $0<r<1$.

Of course $F_{r}, 0<r<1$, approximates $f$ almost uniformly on $M$ as $r \rightarrow 1$. Also using the fact that $\tau(\operatorname{ch}(\bar{V}))$ is polynomially convex, we can approximate $F_{r}$ uniformly on any compact set $K \subset \tau(\operatorname{ch}(\bar{V}))$ by holomorphic polynomials. However, by using Mittag-Leffler type approximations, we can construct the approximating polynomials more explicitly. For functions of one complex variable there is the classical approximation of a holomorphic function in Mittag-Leffler star domains. For details see [M]. We concentrate here mainly on the several variables version of this type of approximation.

There exist universal complex constants

$$
c_{0}^{(n)}, \ldots, c_{k_{n}}^{(n)}, \quad n=0,1, \ldots
$$

such that for any holomorphic function $F_{r}: \Omega_{r} \rightarrow \mathbb{C}$ (the constants are the same for any starlike domain) we have

$$
\begin{aligned}
F_{r}\left(z_{1}, z_{2}\right)= & \sum_{n=0}^{\infty}\left\{\frac{1}{0 !} c_{0}^{(n)} F_{r}(0,0)+\frac{1}{1 !} c_{1}^{(n)}\left[\frac{\partial F_{r}(0,0)}{\partial z_{1}} z_{1}+\frac{\partial F_{r}(0,0)}{\partial z_{2}} z_{2}\right]\right. \\
& \left.+\ldots+\frac{1}{k_{n} !} c_{k_{n}}^{(n)}\left[\sum_{\alpha=0}^{k_{n}}\left(\begin{array}{c}
k_{n} \\
\alpha
\end{array}\right) \frac{\partial^{\alpha} F_{r}(0,0)}{\partial z_{1}^{\alpha}} \frac{\partial^{k_{n}-\alpha} F_{r}(0,0)}{\partial z_{2}^{k_{n}-\alpha}} z_{1}^{\alpha} z_{2}^{k_{n}-\alpha}\right]\right\}
\end{aligned}
$$

and the series converges almost uniformly to $F_{r}$ on the intersection of $\Omega_{r}$ with any one-dimensional complex subspace. Using compactness of the sphere $S^{3}$ whose points give directions of complex subspaces in $\mathbb{C}^{2}$, and the standard arguments, we deduce that the above series converges almost 
uniformly on $\Omega_{r}$. Therefore, its partial sums almost uniformly approximate $F_{r}$, and consequently $f$, on $\Omega_{r}$. The theorem is proved.

Proof of Theorem 0.2. It is enough to consider non-compact manifolds $N$, therefore we have the following two possibilities: $N$ is either an open Jordan arc or an open domain in $\mathbb{R}^{2}$.

The second case is trivial, because then $M$ is an open tube in $\mathbb{C}^{2}$ and by Bochner's tube theorem any holomorphic function on $M$ can be holomorphically extended to the convex hull of $M$ and therefore can be almost uniformly approximated by polynomials.

Consider the first case. Let $N$ be a Jordan arc in $\mathbb{R}^{2}$, smoothly parametrized by $\varphi:(0,1) \rightarrow N$. We can assume that $\operatorname{Int}(\operatorname{ch}(N)) \neq \emptyset$ because otherwise the situation is trivial. For sufficiently small $\delta$ consider the curve $N_{\delta}$ parametrized by $\left.\varphi\right|_{(\delta, 1-\delta)}$. Fix $0<\delta_{0}<1 / 4$ so small that $\operatorname{Int}\left(\operatorname{ch}\left(N_{\delta}\right)\right) \neq$ 0 for $0<\delta<\delta_{0}$. The curve $N_{\delta}, 0<\delta<\delta_{0}$, satisfies the assumptions of Lemmas 3.2 or 3.3. Consequently, using these lemmas we can construct a corresponding curve $C_{\delta}$ which bounds a simply connected domain $W_{\delta}$ and has the properties given by the lemmas. In the case that $N_{\delta}$ satisfies the assumptions of Lemma 3.3, we apply this lemma for $\varepsilon=2 \delta$. Without any loss of generality we can assume that $W_{\delta}$ is starlike with respect to the origin $(0,0)$.

Take any CR function $f: M \rightarrow \mathbb{C}$. Since by the assumptions of the theorem, the curve $N$ has only a finite number of inflexion points on any compact arc, we can apply Kazlow's theorem $[\mathrm{K}]$, and $f$ can be extended to a function $F$ which is smooth on $\operatorname{ach}(M)$ and holomorphic on $\operatorname{Int}(\operatorname{ch}(M))$. By the properties of $C_{\delta},\left.F\right|_{\tau\left(\bar{W}_{\delta}\right)}$ is smooth and holomorphic on $\tau\left(W_{\delta}\right)$. Proceeding in exactly the same way as in the proof of Theorem 0.1, we can approximate $F$ almost uniformly on $\tau\left(\bar{W}_{\delta}\right)$ by holomorphic polynomials which can be chosen as indicated in the mentioned proof.

For any compact set $K \subset M$ there exists $\delta>0$ sufficiently small that $K \subset \tau\left(\bar{W}_{\delta}\right)$ and therefore $f$ can be uniformly approximated on $K$ by holomorphic polynomials.

This completes the proof of Theorem 0.2 .

\section{References}

[AH] A. Andreotii and C. D. Hill, E. E. Levi convexity and the Hans Lewy problem. Part I: Reduction to vanishing theorems, Ann. Scuola Norm. Sup. Pisa 26 (1972), 325-363.

[BT] M. S. Baouendi and F. Trèves, A property of the functions and distributions annihilated by a locally integrable system of complex vector fields, Ann. of Math. 113 (1981), 387-421.

[C] E. M. Chirka, personal communication. 
[D] R. Dwilewicz, Holomorphic approximation in the theory of Cauchy-Riemann functions, in: Complex Analysis, Functional Analysis and Approximation Theory, North-Holland Math. Stud. 125, North-Holland, 1986, 71-82.

[DG] R. Dwilewicz and P. M. Gauthier, Global holomorphic approximations of CR functions on CR manifolds, Complex Variables 4 (1985), 377-391.

[DF] K. Diederich and J. E. Fornæss, Pseudoconvex domains: An example with nontrivial Nebenhülle, Math. Ann. 225 (1977), 275-292.

[FN] J. E. Fornæss and A. Nagel, The Mergelyan property for weakly pseudoconvex domains, Manuscripta Math. 22 (1977), 199-208.

[K] M. Kazlow, CR functions and tube manifolds, Trans. Amer. Math. Soc. 255 (1979), 153-171.

[M] A. I. Markushevich, Theory of Functions of a Complex Variable, Vol. III, Prentice-Hall, Englewood Cliffs, N.J., 1967.

[N1] J. Nunemacher, Approximation theory on totally real submanifolds, Math. Ann. 224 (1976), 129-141.

[N2] - , Approximation theory on CR submanifolds, in: Proc. Sympos. Pure Math. 30, Part II, Amer. Math. Soc., 1977, 181-186.

[S] A. Sakai, Uniform approximation in several complex variables, Osaka J. Math. 15 (1978), 589-611.

DEPARTMENT OF MATHEMATICS

THE UNIVERSITY OF WESTERN ONTARIO

LONDON, ONTARIO, N6A 5B7

CANADA

\author{
INSTITUTE OF MATHEMATICS \\ UNIVERSITY OF WARSAW \\ BANACHA 2 \\ 00-913 WARSZAWA 59
}

POLAND 